\title{
A big thank you to our reviewers in the past year!
}

Good reviewers are essential to the success of any journal and peer review is a major pillar of science. We are grateful to those mentioned below to have dedicated their time and expertise to help our authors improve and refine their manuscripts and support the Editor(s) in the decision making process in the past year. The following received out Top Reviewer of the Year award: Tomonori Yano, Amol Bapaye, Torsten Beyna. We wish all our readers, reviewers, authors and friends a happy and prosperous 2019!

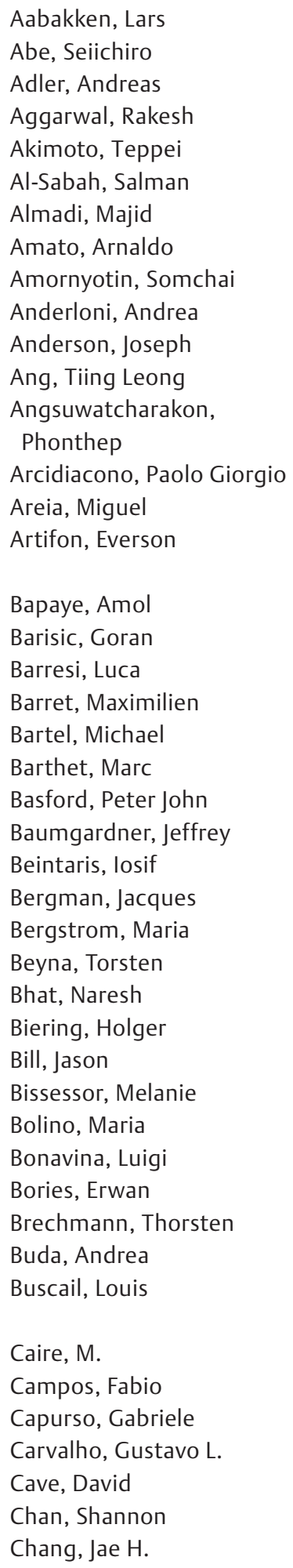

Chen, Shiyao

Chen, Tao

Cheon, Young Koog

Chiba, Hideyuki

Chiu, Han-Mo

Cho, Yu Kyung

Coron, Emmanuel

Costamagna, Guido

Crinò, Stefano Francesco

De La Mora-Levy, Jose G.

de Lange, Thomas

de Moura, Eduardo

De Quadros, Luiz Gustavo

Deprez, Pierre

Desilets, Etienne

Devière, Jacques

Dhir, Vinay

Dias Pereira, Antonio

Diehl, David

Dobru, Daniela

Dohi, Osamu

Dolak, Werner

Doyama, Hisashi

Du, Yiqi

Dumonceau, Jean-Marc

Eglinton, T. W.

El Zein, Mohamad

Elzahry, Mohammad

Emura, Fabian

Etoh, Tsuyoshi

Ezemba, Ndubueze

Fabbri, Carlo

Fábián, Anna

Faiss, Siegbert

Familiari, Pietro

Farrell, James

Ferlitsch, Monika

Fracasso, Pierluigi

Francisco, Ramirez

Freeman, Martin

Frieling, Thomas

Fry, Lucia

Fujihara, Shintaro

Fujii-Lau, Larissa

Fujishiro, Mitsuhiro
Fujiya, Mikihiro

Fusaroli, Pietro

Gangarosa, Lisa

García-Alonso, Francisco

Gawron, Andrew J.

Gen, Yasuyuki

Gleeson, Ferga

Goda, Kenichi

Goto, Osamu

Gralnek, Ian

Greenwald, Bruce

Gromski, Mark

Guo, Yun-Wei

Gurbulak, Bunyamin

Gurvits, G. E.

Gutierrez, Juan

Hakuta, Ryunosuke

Hampe, Jochen

Hardee, Steven

Hashimoto, Rintaro

Hashimoto, Yusuke

Hassan, Cesare

Hatta, Waku

Hirasawa, Kingo

Hisabe, Takashi

Hochberger, Juergen

Hoffman, Arthur

Hori, Keisuke

Horimatsu, Takahiro

Horiuchi, Yusuke

Hosoe, Naoki

Hotta, Kinichi

$\mathrm{Hu}$, Bing

Hyun, Jong Jin

lacopini , Federico

lizuka, Toshiro

Ikematsu, Hiroaki

Imai, Kenichiro

Isayama, Hiroyuki

Ishihara, Ryu

Itoi, Takao
Iwagami, Hiroyoshi

Iwamuro, Masaya

Iwashita, Takuji

Iwatate, Mineo

Jacques, Jeremie

Kaffes, Arthur

Kalmaz, Denise

Kaltenbach, Tonya

Kanamori, Jun

Kanesaka, Takashi

Kanno, Yoshihide

Kanzaki, Hiromitsu

Kashida, Hiroshi

Kato, Motohiko

Kawachi, Hiroshi

Kawakami, Hiroshi

Kawakubo, Kazumichi

Kazuo, Ohtsuka

Khor, Christopher

Kida, Mitsuhiro

Kikuchi, Daisuke

Kim, Jin Hong

Kinugasa, Hideaki

Klapman, Jason

Koay, Doreen

Kobayashi, Masaaki

Kobayashi, Nozomu

Kodashima, Shinya

Kogure, Hirofumi

Komeda, Yoriaki

Kongkam, Pradermchai

Koshy, Abraham

Koulaouzidis, Anastasios

Kown, Yong Hwan

Krishna, Somashekar

Kudo, Shin-ei

Kudo, Toyoki

Kumbhari, Vivek

Kuwai, Toshio

Kwon, Richard S.

Laleman, Wim

Larghi, Alberto

Laugier, Rene

Law, Ryan

Lee, Sang Soo 


\author{
Lee, Tae Hoon \\ Lee, Tsung-Chun \\ Lee, Yun Nah \\ Li, Yan-Qing \\ Lien, Gi-Shih \\ Lisotti, Andrea \\ Liu, Deliang \\ Longcroft-Wheaton, Gaius \\ Lorenzo-Zúñiga, Vicente \\ Lupu, Alexandru
}

Mabe, Katsuhiro

Macedo, Guilherme

Maehata, Tadateru

Maetani, Iruru

Makazu, Makomo

Mangiavillano, Benedetto

Manta, Raffaele

Mark-Christensen, Anders

Martinek, Jan

Mehta, Nilay

Memon, Muhammed

Mikami, Tatsuya

Min, Byung-Hoon

Mion, Francois

Misra, Sri

Mo, Lein-Ray

Moenkemueller, Klaus

Mohamadnejad, Mehdi

Mori, Yuichi

Morita, Flavio

Naganuma, Makoto

Naito, Yuji

Nakai, Yousuke

Nakajima, Atsushi

Nakamura, Masanao

Nakase, Hiroshi

Napoleon, Bertrand

Navaneethan, Udayakumar

Niimi, Keiko

Niv, Yaron

Nonaka, Kouichi

Nonaka, Satoru

Nunobe, Souya
O'Connor, Sam

Ogata, Noriyuki

Ogura, Takeshi

Ohata, Ken

Omar, Artur

Ono, Satoshi

Osawa, Hiroyuki

Othman, Mohamed O.

Pacchierotti, Claudio

Pan, Peng

Pannala, Rahul

Papalois, Apostolos

Papanikolaou, Ioannis

Park, Jung-Hoon

Parra-Blanco, Adolfo

Pennazio, Marco

Perez-Miranda, Manuel

Philip, Mathew

Pineau, Lionel

Pioche, Mathieu

Piraka, Cyrus

Pittayanon, Rapat

Poley, Jan-Werner

Potter, Nancy L.

Probst, Andreas

Puri, Rajesh

Quintero, Enrique

Rai, Praveer

Raja, Kaiser

Rana, Surinder

Rana, Surinder Singh

Reddy, Nageshwar

Reimão, Sílvia

Rosa, Bruno

Sadik, Riadh

Safatle-Ribeiro, Adriana

Sakamoto, Taku

Sanaka, Madhusudhan

Sanders, David

Sandru, Vasile

Saracco, Giorgio

Sasanuma (Hayashi), Seiko
Saurin, Jean-Christophe

Schoefl, Rainer

Seicean, Andrada

Sekiguchi, Masau

Senore, Carlo

Seo, Dong Wan

Sey, Michael

Sgro, Alberto

Shah, Neil

Shah, Raj

Shichijo, Satoki

Shin, Sung Kwan

Siau, Keith

Sidhu, Reena

Singh, Rajvinder

Singla, Vikas

Spada, Cristiano

Stavropoulos, Stavros

Stock, C.

Suzuki, Haruhisa

Suzuki, Sho

Tabibian, James

Takamaru, Hiroyuki

Takenaka, Mamoru

Takeuchi, Yoji

Takizawa, Kohei

Tamai, Naoto

Tanaka, Masaki

Tanaka, Shinji

Tavares, Marta

Teoh, Anthony

Testoni, Pier Alberto

Thosani, Nirav

Toh Yoon, Ezekiel Wong

Topazian, Mark

Toyonaga , Takashi

Traversa, Giampiero

Triantafyllou, Konstantinos

Trindade, Arvind

Tringali, Andrea

Tsuji, Yosuke

Uedo, Noriya

Ueo, Tetsuya

Umar, Muhammad

Upadhyaya, V. D.

Uraoka, Toshio

Uygun, Ibrahim
Valori, Roland

van Halsema , Emo Eise

Varadarajulu, Shyam

Vazquez-Sequeiros, Enrique

Vennalaganti, Prashanth

Voiosu, Theodor

Wagh, Mihir

Walsh, Catharine M.

Watanabe, Masayuki

Waxman, Irving

Wilson, Ana

Xin, Lei

Yamamoto, Katsumi

Yamamoto, Shunsuke

Yamamoto, Takayuki

Yamamoto, Yorimasa

Yamanouchi, Satoshi

Yamasaki, Yasushi

Yamashina, Takeshi

Yamashita, Kentaro

Yamauchi, Hiroshi

Yamaue, Hiroki

Yano, Tomonori

Yoshida, Masao

Yoshida, Naohiro

Yoshida, Naohisa

Yoshii, Shinji

Yoshinaga, Shigetaka

Yoshio, Toshiyuki

Young, C. J.

Yu, Bai

Yung, Diana

Zarate, Angel

Zhan, Tianzuo

Zhang, Shenghong

Zhou, Ping-Hong 